\title{
Do riblets exhibit fully rough behaviour?
}

\author{
Davide Gatti $^{1}$ (1) $\cdot$ Lars von Deyn $^{1} \cdot$ Pourya Forooghi $^{1} \cdot$ Bettina Frohnapfel $^{1}$
}

Received: 4 July 2019 / Revised: 13 February 2020 / Accepted: 15 February 2020 / Published online: 2 March 2020

(c) The Author(s) 2020

\begin{abstract}
Trapezoidal riblets are experimentally investigated in both their drag-reducing and drag-increasing regimes in an air turbulent channel flow facility. The nondimensional riblet spacing $s^{+}$and height $h^{+}$in viscous units are varied by changing the bulk Reynolds number in the range $5 \times 10^{3}<\operatorname{Re}_{b}<8.5 \times 10^{4}$. The change in skin-friction drag is accurately measured by comparing the pressure drop across the test section with smooth and ribbed walls at given values of $\mathrm{Re}_{\mathrm{b}}$. The results show that riblets in the drag-increasing regime behave like typical " $k$ "-type roughness in the fully rough regime only up to $h^{+} \sim 30$, at odds with present understanding. Further increase in $h^{+}$yields a constant relative change of wall shear stress and thus a clear deviation from the fully rough behaviour. The present results are accessible online at the following digital object identifier: 10.5445/IR/1000089978.
\end{abstract}

\section{Graphic abstract}

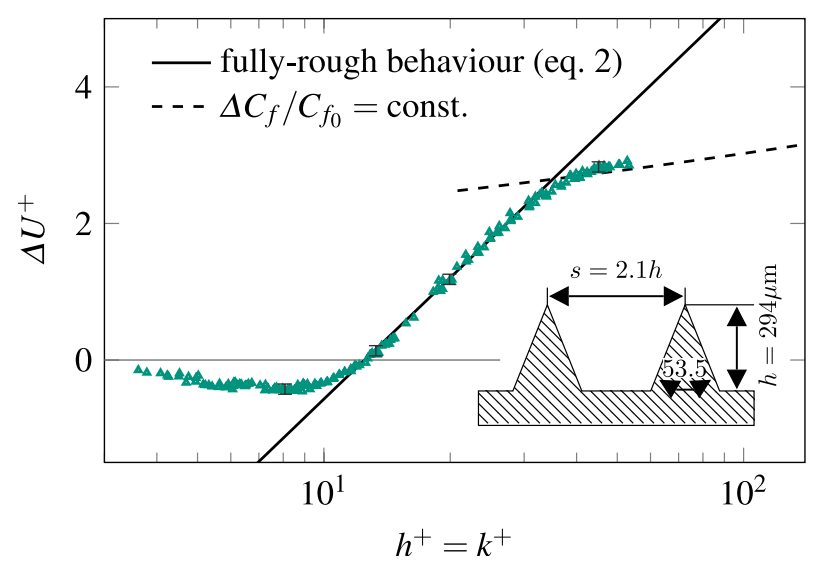

Riblets are small streamwise-aligned ridges that are able to reduce skin-friction drag in turbulent flows. When properly shaped, this anisotropic type of surface roughness results in a lower friction drag than the one found on a smooth wall at the same value of Reynolds number Re.

This work is supported by the Priority Programme SPP 1881 Turbulent Superstructures of the Deutsche Forschungsgemeinschaft.

Davide Gatti

davide.gatti@kit.edu

1 Institute of Fluid Mechanics, Karlsruhe Institute of Technology, Karlsruhe, Germany
The drag-reducing behaviour of riblets has been intensively investigated in the last decades, e.g. by Bechert et al. (1997). For riblets of given aspect ratio, it is known that the spanwise peak-to-peak spacing $s$ of the riblet crests determines the presence and amount of drag reduction. In the limit of vanishing $s$, the drag reduction is proportional to the dimensionless riblet size. In this so-called viscous regime, the drag-reducing mechanism is fairly well understood Luchini et al. (1991) and depends on the riblet ability to oppose cross-flow more than parallel flow, which can be assessed in simple Stokes flows. However, this proportionality breaks down when the riblets spacing scaled in viscous unit $s^{+}=s U_{\tau} / v$ (i.e. made nondimensional by the wall friction velocity $U_{\tau}$ of the flow and the kinematic viscosity $v$ 
of the fluid) becomes sufficiently large. The superscript + indicates nondimensionalisation in viscous units throughout the manuscript.

The breakdown of the viscous regime has been studied numerically by Garcia-Mayoral and Jiménez (2011), who identified the formation of quasi-two-dimensional spanwise vortices as main cause for breakdown and related it to Kelvin-Helmholtz-like instabilities of the mean velocity profile. For sufficiently large values of $s^{+}$, the additional drag due to the two-dimensional spanwise rollers outweighs the drag reduction effect of riblets and the surface exhibits larger skin-friction drag than a smooth wall. Recent numerical studies by Endrikat et al. (2018) suggest that the increase in wall shear stress due to Kelvin-Helmholtz instabilities does not only depend on the riblet size but also on their particular shape.

The drag-increasing regime of riblets is much less studied in the literature Newton et al. (2018); Walsh (1990). The current understanding is that riblets in their drag-increasing regime behave like classical " $k$ "-type roughness, as suggested by Jiménez (2004). For large $k^{+}$, the flow over $k$-type roughness is characterised by the so-called fully rough regime, in which the skin-friction coefficient $C_{f}$ becomes independent of Re and its value is given by an unknown function, determined by the surface geometries, of the relative roughness scale $k / \delta$. Here, $k$ is the roughness length scale and $\delta$ a characteristic length scale of the flow, such as the thickness of a boundary layer or the semi-height of the turbulent channel considered in the following without loss of generality.

The effect of rough surfaces is classically quantified via the downward shift $\Delta U^{+}$of the logarithmic distribution of the mean streamwise velocity profile $U^{+}$in the wall-normal direction $y^{+}$

$U^{+}\left(y^{+}\right)=\kappa^{-1} \log y^{+}+B+G(y / \delta)-\Delta U^{+}$,

which holds in the presence of roughness with the same von Kármán constant $\kappa$ as with smooth walls Jiménez (2004). $B$ is a constant obtained empirically by fitting Eq. 1 with $\Delta U^{+}=0$ against data of turbulent channels with smooth walls. $G(y / \delta)$ is the so-called wake function, which describes the deviation of $U^{+}$from a purely logarithmic behaviour far from the wall. For flows over $k$-type roughness in the fully rough regime, $\Delta U^{+}$, also referred to as roughness function, is related to the roughness scale $k^{+}$through the following logarithmic relationship derived by Perry et al. (1969):

$\Delta U^{+}=\kappa^{-1} \log k^{+}+C$,

where $C$ is a constant, characteristic of the rough surface, that translates $k^{+}$into the equivalent sand grain roughness $k_{s}^{+}$Nikuradse (1933).
Most rough surfaces increase turbulent drag and thus yield a downward shift of the logarithmic portion of $U^{+}\left(y^{+}\right)$ or, in other words, a positive $\Delta U^{+}$. Negative values for $\Delta U^{+}$ can be used to quantify skin-friction drag reduction for those flow control techniques that modify near-wall turbulence in such a way that a mean velocity profile in the form of Eq. 1 is preserved (Gatti and Quadrio 2016). Depending on the operating condition, riblets can yield values of $\Delta U^{+}$of either sign.

The present work addresses the question how $\Delta U^{+}$relates to the dimensionless riblet size $s^{+}$. While the drag-reducing regime is mainly considered for comparison with literature results, the main focus of the present study lies on riblet behaviour past the breakdown of the viscous regime, when riblets act as a drag-increasing roughness. Therefore, an experimental campaign has been carried out in a turbulent channel flow with ribbed walls designed to operate within the drag-reducing and the drag-increasing regime.

The wind tunnel employed is an open-circuit blower tunnel designed by Güttler (2015) for the accurate measurement of small changes in skin-friction drag and has been previously used for measuring drag reduction achieved via spanwise wall oscillations Gatti et al. (2015).

A schematic of the wind tunnel is given in Fig. 1. A centrifugal fan draws air from the environment through an inlet pipe, which houses an orifice flow meter, and directs it against the backside wall of a large settling chamber. In the chamber, air flows at low speeds through a polycarbonate honeycomb flow straightener and five stainless-steel conditioning screens towards the chamber outlet and is accelerated through a fibre-glass nozzle with contraction 6:1 into the test section.

The test section consists of a flat rectangular duct of aspect ratio $\mathrm{AR}=W / H=12$, where $W=300 \mathrm{~mm}$ and $H=25.2 \mathrm{~mm}$ are its inner width and height, respectively. It extends in the streamwise direction for about $3950 \mathrm{~mm}$, corresponding to $157 \mathrm{H}$, and is made up of four independent aluminium parts. Two $15-\mathrm{mm}$-thick aluminium plates constitute the top and bottom walls and are divided into three segments along the streamwise direction, the last

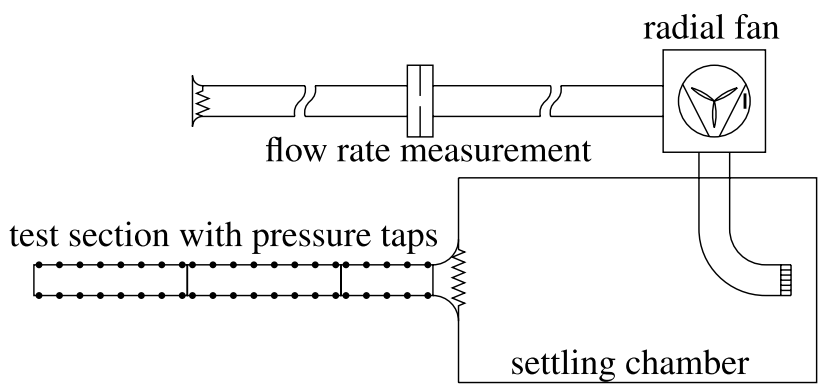

Fig. 1 Schematic of the experimental facility. A detailed description of the facility can be found in Güttler (2015) 
of which is $1500 \mathrm{~mm}$ long and hosts the measurements performed in the present study. All segments are screwed to monolithic side walls, which accurately determines the channel height.

Two trip strips are installed at the inlet of the test section along the whole channel width. Each strip protrudes $1.6 \mathrm{~mm}(0.065 \mathrm{H})$ from the wall and extends for $0.3 \mathrm{~mm}$ in the streamwise direction, resulting in $13 \%$ cross section blockage. The trip provides a fully-developed turbulent flow for a value of the bulk Reynolds number $\operatorname{Re}_{b}=U_{b} H / v$, based on the mean bulk flow velocity $U_{b}$, larger than 4500 and at least $80 H$ past the tripping position. The present measurements are conducted at least $100 H$ downstream of the channel entrance, to exclude any possible uncertainty due to spatial flow development.

Trapezoidal riblets of height $h=294 \mu \mathrm{m}$, spanwise spacing $s=614 \mu \mathrm{m}$ and tip angle $\alpha=53.5^{\circ}$ were milled with micrometre precision into two $1500 \mathrm{~mm} \times 300 \mathrm{~mm}$ polyamide plates in a large milling machine at the Fraunhofer Institute for Production Technology in Aachen, Germany. Bespoke diamond cutting tools were explicitly developed for this purpose. A sketch of the riblet geometry is presented in Fig. 2, while a photograph of the riblet surface can be found in Güttler (2015). The riblet surfaces are mounted onto 15-mm-thick aluminium plates to ensure evenness. These plates are installed as top and bottom walls of the channel in the last segment of the test section. The channel height in this segment is adjusted in such a way that the cross-sectional area $\mathscr{S}$ of the ribbed channel is identical to the one of the smooth channels. Therefore, the average channel height $H=\mathscr{S} / W$ is constant in both set-ups.

In this facility, the present riblet design is expected to yield the largest drag reduction at $\operatorname{Re}_{\mathrm{b}}=11400$. The influence of riblets on skin-friction drag is evaluated throughout the Reynolds number range $5 \times 10^{3}<\mathrm{Re}_{\mathrm{b}}<8.5 \times 10^{4}$ by comparison to the smooth wall reference flow at the same values of $\mathrm{Re}_{\mathrm{b}}$. Such comparison requires the accurate measurement of the wall shear stress $\tau_{w}$, expressed in terms of the skin-friction coefficient

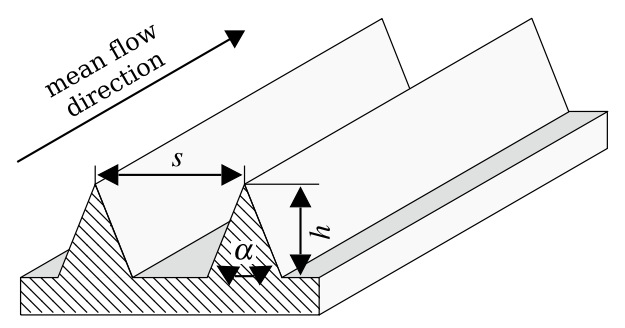

Fig. 2 Schematic sketch of the riblet surface considered in the present study. $s$ and $h$ denote the riblet tip-to-tip spacing and height, respectively, while $\alpha$ is the tip angle
$C_{f}=\frac{2 \tau_{w}}{\rho U_{b}^{2}}$

as well as of the bulk velocity $U_{b}$, fluid density $\rho$ and kinematic viscosity $v$ required for the evaluation of $C_{f}$ and $\mathrm{Re}_{\mathrm{b}}$.

The fluid parameters are determined indirectly via the measurement of ambient pressure, relative humidity and fluid temperature at the inlet and outlet of the wind tunnel, as well as within the settling chamber close to the inlet of the test section. The bulk velocity $U_{b}=\dot{V} /(H W)$ in the channel test section is deduced from the flow rate $\dot{V}$ measured through an orifice flow meter with interchangeable orifice plates using two Setra 239D unidirectional differential pressure transducers with a measurement range up to $125 \mathrm{~Pa}$ and $625 \mathrm{~Pa}$, respectively.

Two MKS Baratron 698A unidirectional differential pressure transducers, with 133 and $1333 \mathrm{~Pa}$ maximum range, measure the head loss along the test section through 21 pairs of pressure taps located at the side walls. Pressure is acquired at all 21 ports in order to verify the streamwise position at which the fully-developed state is achieved.

In the last segment of the test section, where the riblet test plates are installed, the streamwise pressure gradient $\Pi$ is computed by a least-square linear fit of the streamwise evolution of pressure drop measured by 8 consecutive pairs of pressure taps which are each spaced by $200 \mathrm{~mm}$ in the streamwise direction. $\Pi$ is then related to the wall shear stress $\tau_{w}$ through

$\tau_{w}=-0.5 H \Pi$.

According to common practice in the field of riblet drag reduction and roughness, Eq. 4 is utilised for both smooth and ribbed channels, for which $\tau_{w}$ assumes then different meanings. In the former case, $\tau_{w}$ is the actual average friction on the channel walls. In the latter, it assumes the meaning of effective wall shear stress, which is computed by assuming the same reference wetted surface area for ribbed and flat walls, despite the fact that the riblet surface is larger. In the present study, the wetted area of the riblet wall is 1.59 times larger than the flat smooth wall. The wall shear stress defined through Eq. 4 is used to determine the friction velocity $U_{\tau}$.

A detailed description of the facility and the related measurement uncertainties can be found in Gatti et al. (2015); Güttler (2015). Ambient data and the pressure signal at the flow meter are acquired continuously, while the pressure signal along the test section is recorded for $60 \mathrm{~s}$ at each pair of pressure taps, sequentially along the test section via a mechanical multiplexer. To allow pressure lines and sensors to adjust to the new measure, a waiting time of $30 \mathrm{~s}$ is introduced between two consecutive measurements.

The classical way to visualise the drag-reducing performance of riblets is given by the relative change $\Delta C_{f} / C_{f_{0}}$ of 


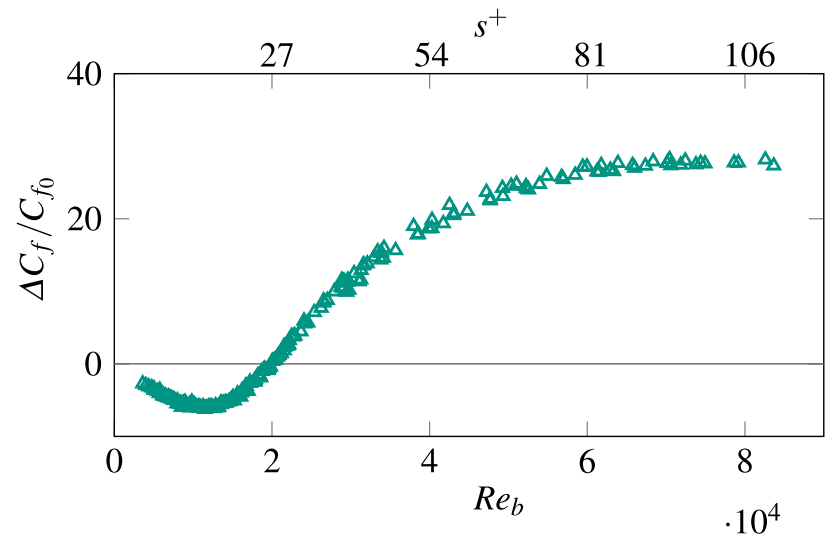

Fig. 3 Relative change of wall shear stress $\Delta C_{f} / C_{f_{0}}$ as function of bulk Reynolds number $\mathrm{Re}_{\mathrm{b}}$ and riblet spacing $s^{+}$in viscous units

skin-friction coefficient $C_{f}$ with respect to the smooth reference $C_{f_{0}}$. The subscript 0 denotes quantities referring to the reference flow over flat smooth surfaces. This quantity is shown in Fig. 3 as a function of $\mathrm{Re}_{\mathrm{b}}$ and the dimensionless riblet spacing $s^{+}$. Drag reduction is found for $\mathrm{Re}_{\mathrm{b}} \leq 20000$ with a maximum drag reduction of $6.2 \%$ at $\mathrm{Re}_{\mathrm{b}}=11459$ which corresponds to $s^{+}=16.4$ and is in very good agreement with literature data Bechert et al. (1997). Beyond $s^{+}=28$, drag is increased compared to the smooth wall reference. The drag further grows for increasing $\operatorname{Re}_{\mathrm{b}}$ but appears to approach a constant value of approximately $28 \%$ for $\mathrm{Re}_{\mathrm{b}}>65000$ or $s^{+}>88$.

Figure 3 also shows that riblets do not behave as $k$-type roughness at large values of $\mathrm{Re}_{\mathrm{b}}$, for which $\Delta C_{f} / C_{f_{0}}$ appears to become constant for the present data. This implies that $C_{f}$ decreases with $\mathrm{Re}$ at a similar rate as $C_{f, 0}$ rather than becoming independent of Re as would occur for a typical $k$-type roughness.

In the following, the observed behaviour of riblets in the rough regime is quantified in terms of the roughness function $\Delta U^{+}$. Since no direct measurement of the mean velocity profile has been performed, $\Delta U^{+}$is estimated from friction and flow rate information as described, for instance, in García-Mayoral and Jiménez (2011); Gatti and Quadrio (2016); Luchini (1996), and summarised in the following.

It is assumed that the logarithmic distribution for $U^{+}\left(y^{+}\right)$ given by Eq. 1 holds with the same $\kappa$ and $G(y / \delta)$ for both flows above flat and ribbed surfaces. Equation 1 is evaluated at the channel centreline for the ribbed and smooth channel. If the latter is subtracted from the former and the result solved for $\Delta U^{+}$, one obtains the following expression:

$\Delta U^{+}=\kappa^{-1} \log \frac{\operatorname{Re}_{\tau}}{\operatorname{Re}_{\tau_{0}}}-\Delta U_{c}^{+}$, which allows to estimate $\Delta U^{+}$provided the friction Reynolds number $\operatorname{Re}_{\tau}=U_{\tau} H / 2 v$ and the centreline velocity $U_{c}$ for both ribbed and smooth channel are known. In the present study, the ribbed and flat surfaces are compared at the same value of $\mathrm{Re}_{\mathrm{b}}$, so that the ratio $\mathrm{Re}_{\tau} / \operatorname{Re}_{\tau_{0}}$ of Eq. 5 will in general not be one.

The direct use of Eq. 5 is not possible for the present set of experiments, in which only $U_{b}$ and not $U_{c}$ was measured. This limitation is circumvented by assuming that $\Delta U_{c}^{+} \approx \Delta U_{b}^{+}$. The approximation is exact for $\operatorname{Re} \rightarrow \infty$, given the initial assumption that the flow over riblets obeys the logarithmic law (Eq. 1) above the buffer layer. Otherwise, it admits an error which depends on the details of how riblets modify the mean velocity profile in the viscous sublayer and buffer layer. Since these details are unknown in the present study, such error is difficult to estimate quantitatively, but it is known to be qualitatively very small in the present Reynolds number range (see, for example, Pope (2000), due to the small size of such layers compared to the channel height and their small contribution to $U_{b}$.

Figure 4 shows the roughness function $\Delta U^{+}$throughout the whole range of $\mathrm{Re}$ investigated in the present study. Since particular interest lies in the existence and persistence of the $k$-type fully rough regime for riblets, the results are reported in the form of Eq. 2, namely representing $\Delta U^{+}$as function of the riblet height $h^{+}$, which is chosen here as roughness length scale $k^{+}$.

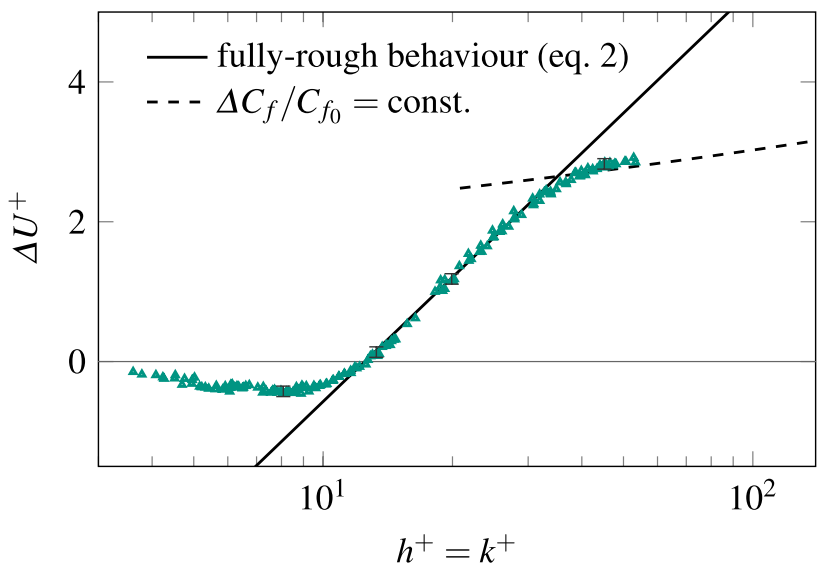

Fig. 4 Downward shift $\Delta U^{+}$of the logarithmic distribution of the mean streamwise velocity profile $U^{+}$against the riblet height $h^{+}$, considered here as roughness scale $k^{+}$. The solid line represents the fully rough behaviour of Eq. 2 with $\kappa=0.39$ and $C=-6.48$, while the dashed line indicates the asymptotic behaviour of $\Delta U^{+}$resulting from Eq. 5 under the assumption that $\Delta C_{f} / C_{f_{0}}=$ const. $=27.5$. The error bars indicate the characteristic uncertainty on $\Delta U^{+}$arising from the arbitrary definition of channel height in the ribbed case, which affects the definition of $\tau_{w}$ in Eq. 4 and thus $U_{\tau}$. This uncertainty is estimated by considering the extreme cases of a virtual wall located at the riblet tip and valley 
As expected, $\Delta U^{+}$is negative in the drag-reducing regime up to $k^{+}=12.5$. Past the breakdown of the viscous regime, which begins at $k^{+} \sim 8, \Delta U^{+}$increases logarithmically with $k^{+}$following a line with slope $\kappa^{-1}$, consistently with the fully rough behaviour for $k$-type roughness of Eq. 2. However, the fully rough regime is not found for indefinitely large $k^{+}$. Starting from $k^{+} \sim 30$, further increasing $k^{+}$yields a lessthan-logarithmic increase in $\Delta U^{+}$and thus results in a clear departure from the fully rough behaviour.

To the authors' knowledge, such departure of riblets from the $k$-type fully rough behaviour has never been reported in literature before. Walsh (1990) collected riblet data in a range of large $k^{+}$similar to the one considered in the present study, albeit for triangular riblets with $h / s>2$ opposed to the present trapezoidal design with $h / s=0.48$. He could not quantify any departure from the fully rough behaviour beyond uncertainty. The same holds for recently reported measurements by Newton et al. (2018). The observed deviation, however, is not fully surprising for riblet surfaces, which do not generate any pressure drag. In fact, the fully rough regime of $k$-type roughness is associated with the share of friction drag becoming smaller compared to that of pressure drag, which instead contributes to $C_{f}$ in a Reindependent fashion.

Figure 3 indicates that $\Delta C_{f} / C_{f_{0}}$ becomes constant at large values of $\operatorname{Re}_{\mathrm{b}}$, i.e. for large $k^{+}$. Since $k^{+}$is changed by varying $\operatorname{Re}_{\mathrm{b}}$ in the present study, such constant $\Delta C_{f} / C_{f_{0}}$ translates into $\Delta U^{+}$slightly increasing with $k^{+}$(see Fig. 4). In this regime, the rate at which $\Delta U^{+}$increases with $k^{+}$can be estimated through Eq. 5 with the approximation $\Delta U_{c}^{+} \approx \Delta U_{b}^{+}$by recalling that $U_{b}^{+}=\sqrt{2 / C_{f}}$ and assuming, for instance, that both $C_{f}$ and $C_{f_{0}}$, which differ by a multiplicative factor, vary with $\mathrm{Re}_{\mathrm{b}}$ via a Dean-type correlation of the form $C_{f} \sim \operatorname{Re}_{\mathrm{b}}^{\gamma}$. The dashed line in Fig. 4 shows the resulting expected behaviour $\Delta U^{+} \sim \operatorname{Re}_{\mathrm{b}}^{-\gamma / 2}$ (and hence $\Delta U^{+} \sim\left(h^{+}\right)^{\frac{\gamma}{\gamma+2}}$ ) for $\gamma=-1 / 4$.

It must be noted that a constant $\Delta C_{f} / C_{f_{0}}$ is compatible with the hydraulic diameter argument Schiller (1923), classically utilised for describing the friction factor in straight ducts with complex cross section. Such argument would hold if large riblets primarily affected the flow by changing the duct contour for the same cross-sectional area while having little impact on local turbulence properties. However, both the classical empirical argument and the recent refinements by Spalart et al. (2018); Pirozzoli (2018) significantly underestimate the value of $\Delta C_{\mathrm{f}} / C_{f_{0}}$ for the present riblet geometry. Similarly, $\Delta C_{f} / C_{f_{0}}$ does not equal the relative increase in surface wetted area. Thus, the change of cross-sectional shape alone does not explain the drag increase by riblets at very large $h^{+}$and other effects may contribute to it, such as the generation of secondary flows which are, however, typically reported for much smaller $h / s$ Goldstein and Tuan (1998).
The intriguing question actually is why riblets demonstrate a $k$-type behaviour at all. A possible explanation could be that the promoters of the fully rough behaviour, which might be the spanwise rollers induced by a Kelvin-Helmholtz instability, are only present within a range of dimensionless riblet spacing $s^{+}$and height $h^{+}$, when the riblets size is compatible with particular turbulent structures. This hypothesis appears to be supported by the fact that in the present experiments, the $k$-type fully rough behaviour incidentally ceases at $h^{+} \sim 30$, when the riblet tip becomes larger than the buffer layer. Whether this speculation reveals true and whether the breakdown of the fully rough regime is present also for different riblet geometries and $h / H$ ratios are still open questions that will be addressed in future studies.

Acknowledgements Open Access funding provided by Projekt DEAL.

Open Access This article is licensed under a Creative Commons Attribution 4.0 International License, which permits use, sharing, adaptation, distribution and reproduction in any medium or format, as long as you give appropriate credit to the original author(s) and the source, provide a link to the Creative Commons licence, and indicate if changes were made. The images or other third party material in this article are included in the article's Creative Commons licence, unless indicated otherwise in a credit line to the material. If material is not included in the article's Creative Commons licence and your intended use is not permitted by statutory regulation or exceeds the permitted use, you will need to obtain permission directly from the copyright holder. To view a copy of this licence, visit http://creativecommons.org/licenses/by/4.0/.

\section{References}

Bechert D, Bruse M, Hage W, Jvd Hoeven, Hoppe G (1997) Experiments on drag-reducing surfaces and their optimization with an adjustable geometry. J Fluid Mech 338:59-87

Endrikat S, Modesti D, García-Mayoral R, Hutchins N, Chung D (2018) Kelvin-Helmholtz rollers in turbulent flow over riblets. In: 21st Australasian fluid mechanics conference, Adelaide, Australia

García-Mayoral R, Jiménez J (2011) Drag reduction by riblets. Phil Trans R Soc A 369(1940):1412-1427

Garcia-Mayoral R, Jiménez J (2011) Hydrodynamic stability and the breakdown of the viscous regime over riblets. J Fluid Mech 678:317-347

Gatti D, Güttler A, Frohnapfel B, Tropea C (2015) Experimental assessment of spanwise-oscillating dielectric electroactive surfaces for turbulent drag reduction in an air channel flow. Exp Fluids 56(5):1-15

Gatti D, Quadrio M (2016) Reynolds-number dependence of turbulent skin-friction drag reduction induced by spanwise forcing. J Fluid Mech 802:553-58

Goldstein DB, Tuan TC (1998) Secondary flow induced by riblets. J Fluid Mech 363:115-151

Güttler A (2015) High accuracy determination of skin-friction differences in an air channel flow based on pressure drop measurement. Ph.D. thesis, Karlsruhe Institute of Technology

Jiménez J (2004) Turbulent flows over rough walls. Annu Rev Fluid Mech 36(1):173-196 
Luchini P (1996) Reducing the turbulent skin friction. In: Desideri et al (eds) Computational methods in applied sciences. Wiley, Hoboken

Luchini P, Manzo F, Pozzi A (1991) Resistance of a grooved surface to parallel flow and cross-flow. J Fluid Mech 228:87-109

Newton R, Chung D, Hutchins N (2018) An experimental investigation into the breakdown of riblet drag reduction at post-optimal conditions. In: 21st Australasian fluid mechanics conference, Adelaide, Australia

Nikuradse J (1933) Strömungsgesteze in Rauhen Rohren. VDI Forsch. 361. (Engl. Trans. 1950. Laws of flow in rough pipes. NACA TM 1292)

Perry A, Schofield W, Joubert P (1969) Rough wall turbulent boundary layers. J Fluid Mech 37:383-413

Pirozzoli S (2018) On turbulent friction in straight ducts with complex cross-section: the wall law and the hydraulic diameter. J Fluid Mech 846:R1

Pope S (2000) Turbulent flows. Cambridge University Press, Cambridge
Schiller L (1923) Über den Strömungswiderstand von Rohren verschiedenen Querschnitts und Rauhigkeitsgrades. Z Angew Math Mech 3(1):2-13

Spalart PR, Garbaruk A, Stabnikov A (2018) On the skin friction due to turbulence in ducts of various shapes. J Fluid Mech 838:369-378

Walsh MJ (1990) Riblets. In: Bushnell DM, Hefner JN (eds) Viscous drag reduction in boundary layers. Progress in astronautics and aeronautics, vol 123, pp 203-261. https://doi.org/10.2514/4.86597 8

Publisher's Note Springer Nature remains neutral with regard to jurisdictional claims in published maps and institutional affiliations. 